\title{
Force-Torque Sensor Disturbance Observer using Deep Learning
}

\author{
Kamal Mohy el Dine ${ }^{\star}$,Jose Sanchez ${ }^{\star}$, Juan Antonio Corrales, Youcef Mezouar, \\ and Jean-Christophe Fauroux \\ Université Clermont Auvergne, SIGMA Clermont, Institut Pascal BP 10448, F-63000 \\ Clermont-Ferrand, France \\ Corresponding author: kamal.mohy_el_dine@sigma-clermont.fr
}

\begin{abstract}
Robots executing force controlled tasks require accurate perception of the applied force in order to guarantee precision. However, dynamic motions generate non-contact forces due to the inertia. These non-contact forces can be regarded as disturbances to be removed such that only the forces generated by contacts with the environment remain. This paper presents an observer based on a recurrent neural network that estimates the non-contact forces measured by a force-torque sensor attached at the end-effector of a robotic arm. The approach is proven to also work with an external load attached to the robotic arm. The recurrent neural network observer uses signals from the joint encoders of the robotic arm and a low-cost inertial measurement unit to estimate the wrenches (i.e. forces and torques) generated due to gravity, inertia, centrifugal and Coriolis forces. The accuracy of the proposed observer is experimentally evaluated by comparing the measurements of the attached force-torque sensor to the observer's non-contact forces estimation. Additionally, the pure contact force estimation is evaluated against an external force-torque sensor.
\end{abstract}

\section{Introduction}

In order for robots to perform tasks involving physical interactions in unknown and dynamic environments, such as physical human-robot collaboration, haptic control (e.g. bilateral teleoperation) and locomotion; it is necessary that they accurately and timely perceive contacts with the environment. Thus, estimation of external contact forces is required to guarantee the success of robotic tasks where contacts are present. In order to estimate contact forces, force-torque sensors are usually attached to the robot's end-effector to measure wrenches generated while interacting with the environment. However, as the force-torque sensor measures both internal and external forces, it is necessary to first estimate the internal (non-contact) forces caused by gravity, inertia, Coriolis and centrifugal forces. Once these non-contact forces have been estimated, they can be then subtracted from the force-torque sensor output to obtain the pure external (contact) forces, as shown in the block diagram in Figure 1b.

* These authors contributed equally to the work. 


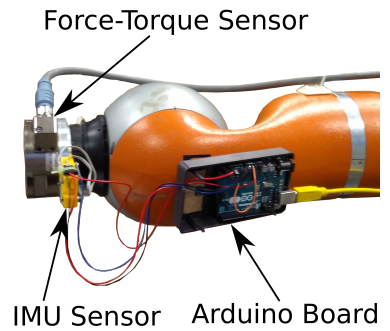

(a) Experimental setup showing the force-torque sensor and the IMU mounted on a KUKA robot.

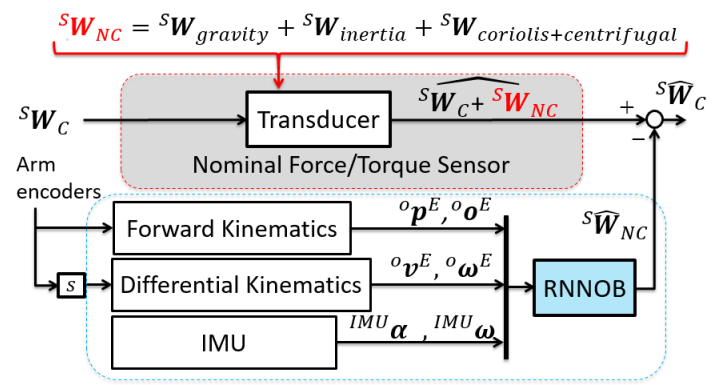

(b) Recurrent neural network observer to estimate the non-contact forces ( $o$ : robot frame, $E$ : end-effector frame, $S$ : sensor frame, $I M U$ : IMU frame).

Fig. 1: Experimental setup and our proposed force observer.

Several works have focused on estimating non-contact forces on a force-torque sensor by incorporating acceleration signals. For instance, García et al. used an observer based on a state-space system that included the dynamics of the robot [1]. Equations based on the inertia matrix of a known load attached to the force-torque sensor were used in [2] to estimate non-contact forces. Similarly, Kubus and Wahl estimated the inertial parameters of the attached load by identification, then the corresponding internal forces were calculated using the Newton-Euler formulation [3].

Instead of requiring accurate estimation of parameters such as the inertia matrix, mass and the center of mass position, other approaches have relied on machine learning methods to directly estimate forces. One of the first works in robotics to use neural networks for estimating forces, was described in [4], where a feedforward neural network approximated two-dimensional forces based on the robot's joint positions, velocities and accelerations. More recently, Kollmitz et al. estimated a six-dimensional contact force on a robot platform using a timedelay neural network [5], where the network delayed the inputs (wrench and acceleration measurements) in order to include temporal information. Another network architecture that makes use of sequential data are recurrent neural networks (RNNs). This type of networks has been successfully applied to estimate forces in robotic tasks. For instance, a force distribution map on a person's limb generated by contacts with a hospital gown was estimated in [6] and contact transients during a snap-fit assembly task were detected in [7]. Based on the recent success in applying RNNs to estimate forces in robotic tasks, we propose to train a recurrent neural network using the robot's proprioceptive information and a low-cost accelerometer to estimate directly the non-contact forces. 


\section{Technical Approach}

The wrench output of the force-torque sensor can be expressed as:

$$
\boldsymbol{W}=\boldsymbol{W}_{N C}+\boldsymbol{W}_{C}=[\boldsymbol{F}, \boldsymbol{\Gamma}]^{T}
$$

where $\boldsymbol{W}_{N C}=\left[\boldsymbol{W}_{\text {gravity }}, \boldsymbol{W}_{\text {inertia }}, \boldsymbol{W}_{\text {coriolis+centrifugal }}\right]^{T}$ is the disturbance wrench affecting the sensor due to the non-contact forces and torques $\boldsymbol{f}_{N C}$ and $\boldsymbol{\tau}_{N C}$ respectively. $\boldsymbol{W}_{C}$ is the pure contact wrench due to contact forces and torques $\boldsymbol{f}_{C}$ and $\boldsymbol{\tau}_{C}$ respectively. $\boldsymbol{F}$ and $\boldsymbol{\Gamma}$ are the force and torque values expressed in the sensor frame $S$ respectively as:

$$
\begin{aligned}
& \boldsymbol{F}=\boldsymbol{f}_{N C}+\boldsymbol{f}_{C} \\
& \boldsymbol{\Gamma}=\boldsymbol{\tau}_{N C}+\boldsymbol{\tau}_{C}
\end{aligned}
$$

Using the Newton-Euler approach, $\boldsymbol{f}_{N C}$ and $\boldsymbol{\tau}_{N C}$ can be expanded and equations (2) and (3) can be rewritten as:

$$
\begin{gathered}
\boldsymbol{F}=m \boldsymbol{\alpha}-m \boldsymbol{g}+\dot{\boldsymbol{\omega}} \times m \boldsymbol{c}+\boldsymbol{\omega} \times(\boldsymbol{\omega} \times m \boldsymbol{c})+\boldsymbol{f}_{C} \\
\boldsymbol{\Gamma}=\boldsymbol{I} \dot{\boldsymbol{\omega}}+\boldsymbol{\omega} \times(\boldsymbol{I} \boldsymbol{\omega})+m \boldsymbol{c} \times \boldsymbol{\alpha}-m \boldsymbol{c} \times \boldsymbol{g}+\boldsymbol{\tau}_{C}
\end{gathered}
$$

where $\boldsymbol{\omega}$ is the angular velocity vector of the sensor with respect to its frame, $\boldsymbol{\alpha}$ and $\dot{\boldsymbol{\omega}}$ are the linear and angular acceleration vectors respectively, $\boldsymbol{g}$ is the vector corresponding for gravity, $m$ is the mass of the load, $\boldsymbol{c}$ is its center of mass coordinates vector and $\boldsymbol{I}$ is a $3 \times 3$ symmetric matrix representing the inertia matrix in the sensor frame.

In standard control approaches, when accuracy is not critical, the sensor measurements in equations (2) and (3) are used in their default form taking both contact and noncontact forces as the feedback signal to the controller. However, when accurate force control is required, non-contact forces need to be removed as they can lead to wrong reference force values fed back to the controller. In order to estimate these forces precisely, the ten inertial parameters of the load should be known, namely: $m, \boldsymbol{c}$ and the values of $\boldsymbol{I}$. In the literature, researchers usually use identification methods to obtain these values and apply equations (4) and (5) to calculate the non-contact forces and torques. However, the accuracy of estimating non-contact forces and torques based on identification is dependent on the accuracy of the center of mass position of the load $\boldsymbol{c}$ and the calculation of the kinematic vectors $\boldsymbol{\alpha}, \boldsymbol{\omega}$ and $\dot{\boldsymbol{\omega}}$ in the same frame.

To overcome these inaccuracies, we propose an observer based on a recurrent neural network (RNNOB) to estimate directly non-contact forces independently of the twist and acceleration transformations. Since the involved signals are sequential, an RNN architecture using Long Short-term Memory (LSTM) units, as described in [8], is ideal to correlate the sensor's kinematics to its wrench output. Figure 1 shows the sensors mounted on the arm along with block diagram of the RNNOB showing how the non-contact forces are estimated and then canceled from the force-torque sensor measurements. 


\section{Experiments: Data Collection and Testing}

As shown in equations (4) and (5), the non-contact forces are directly related to angular velocities and accelerations, linear accelerations and the sensor orientation (due to gravity effects). Thus, in order to train the observer it is necessary to generate a broad spectrum of states for these variables. To this end, two datasets were collected using the joint encoders of a KUKA LWR-4 arm with the ATI Gamma ${ }^{1}$ force-torque sensor and an Adafruit (L3GD20H + LSM303) ${ }^{2}$ inertial measurement unit (IMU) mounted on its wrist. The first dataset was collected without an external load (see Figure 1a), while the second had the Shadow Dexterous Hand ${ }^{3}$ attached as an external load, as shown in Figure 2. The measurement and average rates for the sensors used, as depicted in Figure 1, are described below:

1. ATI Gamma: produces a six-dimensional wrench expressed in the sensor's frame $S$ at $1,000 \mathrm{~Hz}$.

2. Adafruit (L3GD20H + LSM303): generates linear accelerations and angular velocities expressed in the $I M U$ frame at $300 \mathrm{~Hz}$.

3. Joint encoders: Provide, through forward and differential kinematics, the end-effector orientation (in quaternion representation) plus linear and angular velocities expressed in the robot frame $\Sigma_{O}$ at $500 \mathrm{~Hz}$.

Since the sensors operate at different rates, the data was recorded at 500 $\mathrm{Hz}$ to have a uniform sampling rate. Thus, the force-torque sensor is effectively downsampled and the last output of the IMU is kept until a new sample is published.

\subsection{Data collection without load}

For this dataset, the data collected was generated by manual and automatic trajectories. The manual data was collected by setting the robot controller to gravity compensation mode and then moving the wrist manually to various poses in the workspace with random velocities and accelerations. The automatic data (e.g. without human intervention) was generated by moving the robot between random points in its workspace using various trapezoidal velocity profiles. The manual data was collected in six trials, each about four minutes long and the automatic data was collected in ten trials with an average time of two minutes each. These datasets were then combined into one training dataset, where one trial of each of the manual and automatic data were separated to create a test dataset. The validation of the RNN during training used $20 \%$ of the training dataset.

\footnotetext{
${ }^{1}$ http://www.ati-ia.com/products/ft/ft_models.aspx?id=Gamma

${ }^{2}$ https://www.adafruit.com/product/1714

${ }^{3}$ https://www.shadowrobot.com/products/dexterous-hand/
} 


\subsection{Data collection with external load}

Besides the manual and automatic trajectories, described in subsection 3.1, an additional sinusoidal trajectory on the $x y$-plane of the robot (see Figure 2) frame was generated for this dataset. During this sinusoidal trajectory the end-effector was rotated around each axis sequentially (i.e. roll, pitch and then yaw).

The manual data was collected in five trials, each about five minutes long; the automatic data was collected in ten trials with an average time of two minutes each, and the sinusoidal data was collected in one trial about three minutes long. These datasets were then combined into one training dataset, where one trial of each of the manual and automatic data were separated to create a test dataset. The sinusoidal data was used entirely for training. The validation of the RNN during training used $20 \%$ of the training dataset. Additionally to the testing set described above, a pure rotational motion test was used to validate the proposed observer against gravitational forces. This rotational test can be seen in Figure 7, where the angular rotations are shown at the bottom of the figure.

\subsection{Collision test}

To show the accuracy of the proposed approach in estimating pure contact forces, an ATI Mini $45^{4}$ force-torque sensor was used as reference. The arm was set to gravity compensation mode to move the external load and collide it ten times with the reference force-torque sensor fixed on the table as shown in Figure 2.

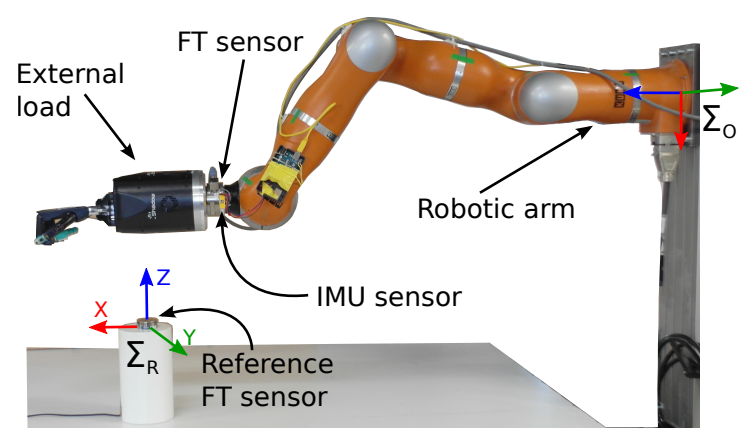

Fig. 2: Collision test setup.

\footnotetext{
${ }^{4}$ https://www.ati-ia.com/products/ft/ft_models.aspx?id=Mini45
} 
Kamal Mohy el Dine et al.

\section{Results}

\subsection{RNN model}

To validate our RNN observer, we trained different models using the position $(p)$, orientation $(o)$ and the twist $(v, \omega)$ derived from the joint encoders ${ }^{5}$; and the linear acceleration obtained from the IMU $\left({ }^{I M U} \alpha\right)$. Besides different input features, models with different hyperparameters such as the number of epochs, sequence length and learning rate, were also tested. However, the difference in performance between these networks was not statistically noticeable. Thus, the three different models based on the pose $(p, o)$, orientation and twist $(o, v, \omega)$, and linear acceleration, orientation and twist $\left({ }^{I M U} a, o, v, \omega\right)$ were evaluated. All these models were trained for 20 epochs using an architecture with two hidden layers, with 15 and 10 LSTM units respectively, and a sequence length for the input layer of 20 time steps (0.2 seconds). In the output layer, we applied Stochastic Gradient Descent with a learning rate of 0.01 to minimize the mean square error of the regression problem. A hyperbolic tangent sigmoid function was used as the activation function between the hidden layers and for the output layer a linear activation function was used.

Figure 3 shows the performance achieved by the models based on different input features on the dataset with an external load as described in Section 3.2. It can be clearly seen that the model incorporating the measurements from the IMU $\left({ }^{I M U} a, o, v, \omega\right)$ achieves the best overall performance. However, for the rotation test, as the accelerations are small and only the orientation changes, this model is slightly outperformed by the one using only pose information $(p, o)$.

\subsection{Force estimation without load}

The root mean square (RMS) errors on the test datasets of the automatic and manual motions without load, as described in Section 3.1, are summarized in Table 1. This table shows the force estimation of the RNN observer for previously unseen automatic and manual trajectories compared to the measurement of the force-torque sensor. Since no load was attached to the sensor, except for the top plate which has a negligible mass, the generated wrenches remained very low. The force values were under $\pm 1.2 \mathrm{~N}$ while the torques did not exceed $\pm 0.6 \mathrm{~N} \cdot \mathrm{m}$ (see Figure 4).

\subsection{Force estimation with external load}

To compare the performance of the proposed observer, we implemented an analytical observer using the method described in [3]. As this method requires knowledge of the inertial parameters, we estimated these parameters using least squares on a trial of the manual dataset (described in Section 3.2). Both methods were compared against the wrenches measured by the force-torque sensor

\footnotetext{
${ }^{5}$ Note that for simplicity, when the subscripts are not indicated the quantities are assumed to be of the end-effector expressed on the robot frame.
} 


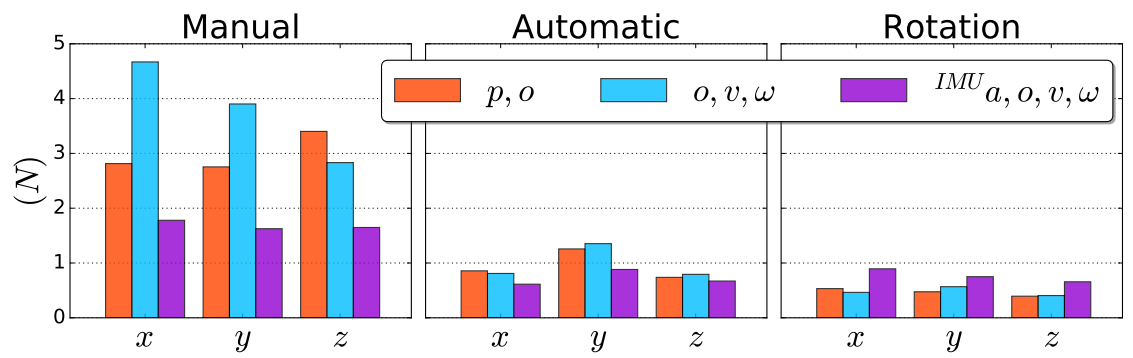

(a) Linear errors for the three types of test motions.

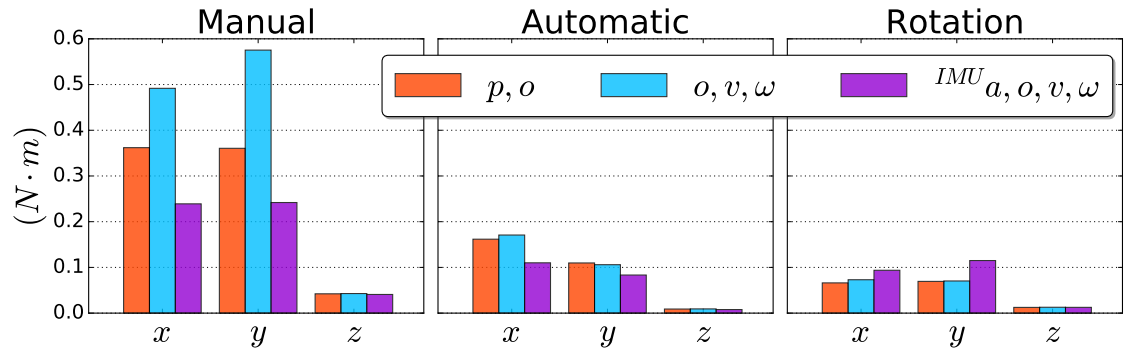

(b) Angular errors for the three types of test motions.

Fig. 3: RMS errors for the RNN models based on: 1) pose $(p, o), 2)$ orientation and twist $(o, v, \omega)$ and 3$)$ linear acceleration, orientation and twist $\left({ }^{I M U} a, o, v, \omega\right)$.

and their corresponding RMS errors are shown in Table 2. These results were obtained for the three motion tests described in Section 3.2, namely, manual, automatic and rotational. A visual example of the force estimation for both, RNN and analytical, observers against the measured forced for a previously unseen manual trajectory can be seen in Figure 5.

Additionally, the efficiency of these methods in estimating gravitational forces was evaluated by the rotational test as it can be seen in Figure 7. Table 2 clearly shows that the proposed RNNOB outperforms the analytical observer in all motion tests.

\subsection{Collision test}

The mean error for the ten collisions was of $1.197 \pm 0.338 N$. One example of the contact tests is shown in Figure 6, the contact force is expressed in the reference sensor frame on the table. It can be seen from the figure that the proposed observer can estimate the contacts that are larger than $2 N$. 
Table 1: RMS errors on the automatic and manual test datasets without an external load.

\begin{tabular}{c|c|c} 
& \multicolumn{2}{c}{ Automatic } \\
\cline { 2 - 3 } & $N$ & $N \cdot m$ \\
\hline $\mathbf{X}$ & 0.0432 & 0.0010 \\
$\mathbf{Y}$ & 0.0380 & 0.0007 \\
$\mathbf{Z}$ & 0.0268 & 0.0009
\end{tabular}

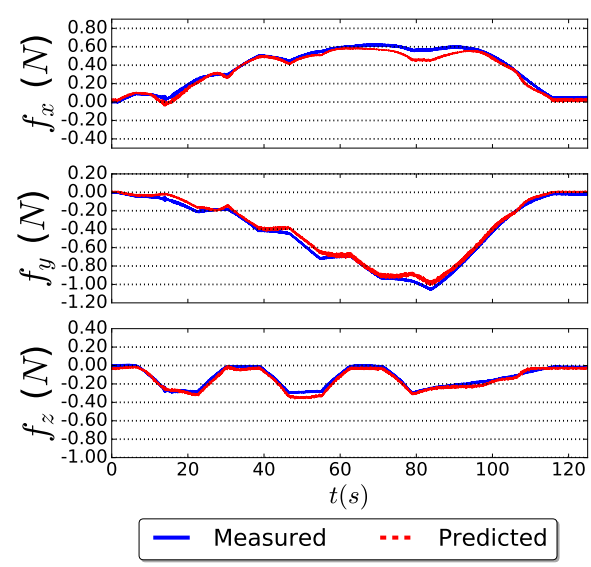

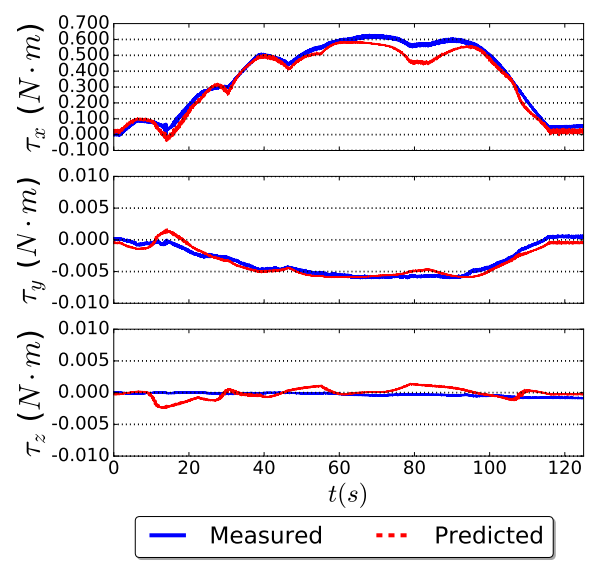

Fig. 4: Non-contact forces $\left({ }^{S} \widehat{\boldsymbol{W}}_{N C}\right)$ estimated by the RNNOB for an unseen automatic trajectory with no load attached to the force-torque sensor. The yaxis shows the forces and torques as measured in the force-torque sensor frame.

Table 2: The root mean square error on the dataset with an external load for the proposed RNNOB and the analytical method based on identification.

\begin{tabular}{c|c|c|c|c|c|c|c}
\multicolumn{2}{c}{} & \multicolumn{2}{c|}{ Manual } & \multicolumn{2}{c|}{ Automatic } & \multicolumn{2}{c}{ Rotational } \\
\cline { 2 - 7 } \multicolumn{2}{c}{} & RNNOB & Analytical & RNNOB & Analytical & RNNOB & Analytical \\
\hline \multirow{2}{*}{ Error } & $f_{x}$ & 1.6578 & 2.8934 & 0.8075 & 2.4436 & 0.8405 & 2.6900 \\
$(N)$ & $f_{y}$ & 1.7235 & 2.6943 & 1.1334 & 2.8558 & 0.7189 & 1.2193 \\
& $f_{z}$ & 1.5420 & 2.2989 & 0.7829 & 1.5147 & 0.7082 & 1.2204 \\
\hline \multirow{2}{*}{ Error } & $\tau_{x}$ & 0.2538 & 0.3358 & 0.1384 & 0.2835 & 0.0911 & 0.1408 \\
$(N \cdot m)$ & $\tau_{y}$ & 0.2332 & 0.3420 & 0.0995 & 0.2020 & 0.1053 & 0.1601 \\
& \multirow{2}{*}{$* 0.0403$} & 0.0513 & 0.0089 & 0.0132 & 0.0118 & 0.0301
\end{tabular}




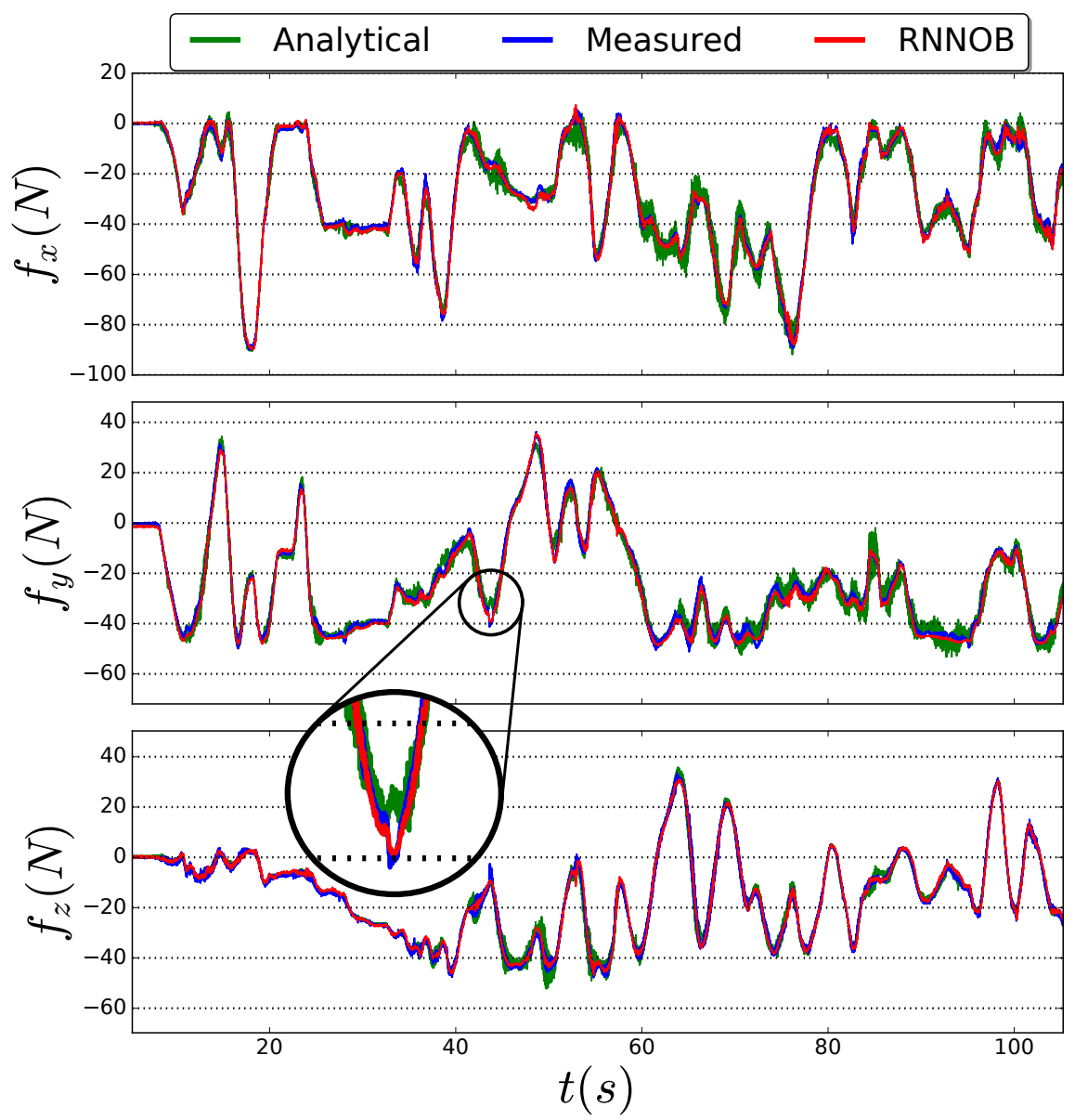

Fig. 5: Non-contact wrench estimation of the proposed RNNOB compared with an analytical-based approach and as measured by the force-torque sensor for an unseen manual trajectory with an external load. The $y$-axis shows the forces along $x, y$ and $z$ as measured in the force-torque sensor frame respectively. 


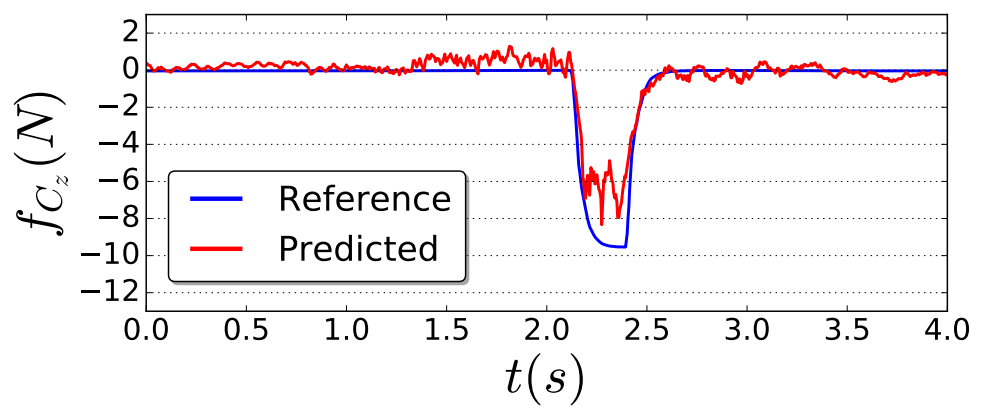

Fig. 6: Contact force estimation of the proposed approach compared to the reference force measurement.

\section{Conclusions}

The article presented a recurrent neural network observer that estimates the noncontact forces acting on the force-torque sensor, namely: inertial, gravitational, centrifugal and Coriolis forces. The proposed observer is model-free and was able to accurately estimate the non-contact forces when the force-torque sensor is subject to high dynamic motions without the need of an identification process. Additionally, in a variety of highly dynamic motions, the RNNOB outperformed the analytical method based on the identification of the inertial parameters. Furthermore, the input signals used by the proposed observer were unfiltered and thus, no additional delay is introduced. Besides, pure contact forces can be obtained by subtracting the output of observer from the output of the forcetorque sensor such that external contacts can be detected.

The observer is limited by its inability to estimate zero forces when the robot is motionless. This is a known issue of RNNs and some work has been proposed to alleviate this problem, as suggested in [6]. Another interesting future improvement would be to combine the analytical observer with the RNNOB to address the shortcomings of our proposed observer, as well as addressing safety issues by relying on a model-based approach.

\section{Acknowledgements}

Kamal Mohy El Dine works for the European project Robots to Re-Construction (Bots2ReC). His research leading to these results has received funding from the European Union's Horizon 2020 research and innovation programmes under grant agreement No. 687593.

Jose Sanchez is sponsored by the French government research program Investissements d'Avenir through the RobotEx Equipment of Excellence (ANR-10-EQPX44) and the IMobS3 Laboratory of Excellence (ANR-10-LABX-16-01), by the European Union through the program Regional competitiveness and employment 2007-2013 (ERDF - Auvergne region) and by the Auvergne region. 

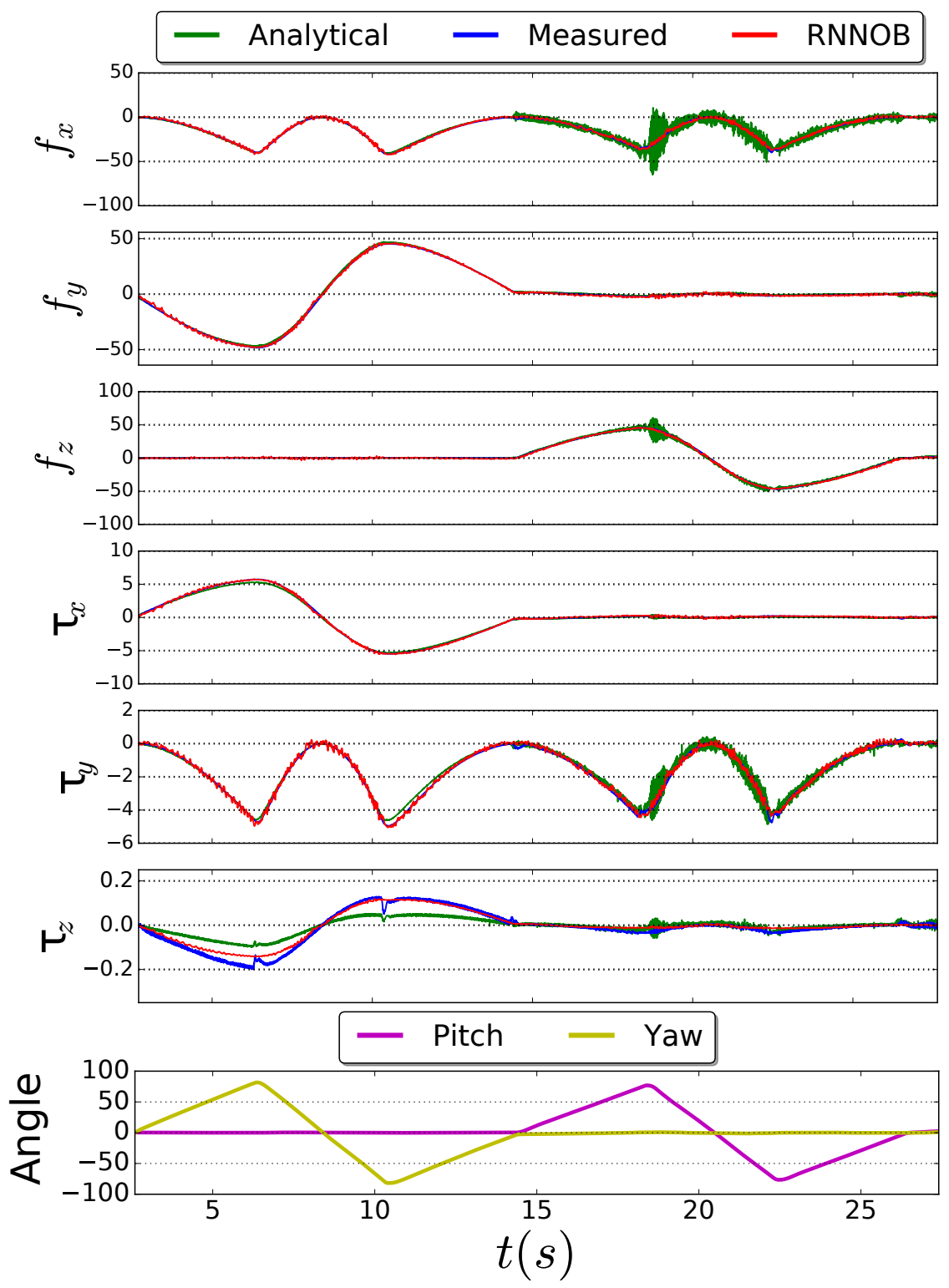

Fig. 7: Non-contact wrench estimation of the proposed RNNOB compared with an analytical-based approach and as measured by the force-torque sensor. The wrench was generated by rotating the end-effector, with an attached load, around the roll, pitch and yaw axes to experience gravitational forces along the three axis of the sensor. The first three rows show the forces in the $x, y$ and $z$ axes of the force-torque sensor frame and are expressed in $N$. The next three rows show the torques and are expressed in $N \cdot m$. The last row shows the rotations around the sensor's $y$-axis (pitch) and $z$-axis (yaw) expressed in degrees. The roll angle is not shown since it has no significant effect as the sensor's $x$-axis is along the gravity vector $\boldsymbol{g}$. 


\section{References}

1. Javier Gámez García, Anders Robertsson, Juan Gómez Ortega, and Rolf Johansson. Force and Acceleration Sensor Fusion for Compliant Robot Motion Control. In IEEE International Conference on Robotics and Automation, pages 2709-2714, Barcelona, Spain, 2005.

2. Torsten Kröger, Daniel Kubus, and Friedrich Wahl. 6D Force and Acceleration Sensor Fusion for Compliant Manipulation Control. In IEEE/RSJ International Conference on Intelligent Robots and Systems, pages 2626-2631, Beijing, China, 2006.

3. Daniel Kubus and Friedrich M. Wahl. Scaling and eliminating non-contact forces and torques to improve bilateral teleoperation. In IEEE/RSJ International Conference on Intelligent Robots and Systems, pages 5133-5139, St. Louis, USA, 2009.

4. Andrew C. Smith, Farid Mobasser, and Keyvan Hashtrudi-Zaad. Neural-networkbased contact force observers for haptic applications. IEEE Transactions on Robotics, 22(6):1163-1175, 2006.

5. Marina Kollmitz, Daniel Büscher, Tobias Schubert, and Wolfram Burgard. WholeBody Sensory Concept for Compliant Mobile Robots. In (To appear in) IEEE International Conference on Robotics and Automation, Brisbane, Australia, 2018.

6. Zackory Erickson, Alexander Clegg, Wenhao Yu, Greg Turk, C. Karen Liu, and Charles C. Kemp. What does the person feel? Learning to infer applied forces during robot-assisted dressing. In IEEE International Conference on Robotics and Automation, pages 6058-6065, Singapore, 2017.

7. Martin Karlsson, Anders Robertsson, and Rolf Johansson. Detection and Control of Contact Force Transients in Robotic Manipulation without a Force Sensor. In IEEE International Conference on Robotics and Automation, pages 21-25, Brisbane, Australia, 2018.

8. Alex Graves, Abdel-rahman Mohamed, and Geoffrey Hinton. Speech recognition with deep recurrent neural networks. In IEEE International Conference on Acoustics, Speech and Signal Processing, pages 6645-6649, Vancouver, Canada, 2013. 